\title{
EchoGéo
}

$44 \mid 2018$

Les valorisations territoriales et touristiques du street art

\section{Le street art entre valorisation informelle du territoire et logiques d'institutionnalisation}

Le cas du projet des Oides à Saint-Nazaire

\section{Aude Le Gallou}

\section{(2) OpenEdition Journals}

\section{Electronic version}

URL: https://journals.openedition.org/echogeo/15564

DOI: 10.4000/echogeo.15564

ISSN: 1963-1197

\section{Publisher}

Pôle de recherche pour l'organisation et la diffusion de l'information géographique (CNRS UMR 8586)

\section{Electronic reference}

Aude Le Gallou, "Le street art entre valorisation informelle du territoire et logiques

d'institutionnalisation", EchoGéo [Online], 44 | 2018, Online since 31 July 2018, connection on 31 July 2021. URL: http://journals.openedition.org/echogeo/15564 ; DOI: https://doi.org/10.4000/echogeo. 15564

This text was automatically generated on 31 July 2021.

EchoGéo est mis à disposition selon les termes de la licence Creative Commons Attribution - Pas d'Utilisation Commerciale - Pas de Modification 4.0 International (CC BY-NC-ND) 


\title{
Le street art entre valorisation informelle du territoire et logiques d'institutionnalisation
}

\author{
Le cas du projet des Oides à Saint-Nazaire
}

\author{
Aude Le Gallou
}

\section{Introduction}

1 «Saint-Nazaire : Le street art envahit la ville». C'est ainsi que le quotidien local Presse Océan décrivait, à la fin de l'été 2017 , la multiplication des œuvres d'art urbain sur les murs de cette ville portuaire du littoral atlantique ${ }^{1}$. Longtemps associée à une image de ville industrielle en crise, Saint-Nazaire connaît aujourd'hui une progressive réhabilitation dans l'imaginaire collectif. Aux yeux des acteurs locaux, l'apparition récente du street art dans l'espace urbain constitue un signe de ce renouveau: responsables institutionnels, commerçants et habitants saluent ainsi des initiatives qui «font danser les murs de la ville $»^{2}$ (Saint-Nazaire Agglomération Tourisme).

2 Si son affirmation dans l'espace public n'en est qu'à ses débuts à Saint-Nazaire, le street art est déjà activement promu par les conseils municipaux de nombreuses métropoles occidentales. De plus en plus visible sur les façades urbaines, il fait également l'objet d'un intérêt croissant des recherches en sciences sociales. Alors que plusieurs chercheurs ont souligné le rôle de l'art et des artistes dans les processus de construction de l'espace urbain (Grésillon, 2002, 2014 ; Guinard, 2014), le street art est un objet de recherche relativement récent. Les travaux qui s'en saisissent analysent la progressive institutionnalisation de la pratique (Genin, 2013) tout comme sa construction progressive en tant qu'objet de gouvernement urbain au croisement de plusieurs catégories d'action publique (Vaslin, 2017) ${ }^{3}$, ainsi que sa mobilisation par les politiques urbaines dans un but de valorisation du territoire dans le cadre de logiques événementielles (Sallenave, 2017) ou comme mode de valorisation des projets urbains (Kullmann, 2017). Certains chercheurs soulignent les ambiguïtés de la réévaluation de 
la sous-culture du graffiti, qui accompagne souvent la promotion du modèle de la ville créative, et esquissent une géographie morale des pratiques créatives (McAuliffe, 2012). D'autres mettent en évidence l'intérêt du street art pour analyser les enjeux relatifs à l'urbanisme sécuritaire et au contrôle croissant propres à la ville néo-libérale (Iveson, 2010). En tant qu'objet de recherche, le street art permet ainsi d'analyser des dynamiques urbaines globales et d'interroger la production contemporaine de l'espace urbain.

3 À la suite de Pauline Guinard, Sébastien Jacquot et Clotilde Kullmann, nous envisageons le street art comme un ensemble de "pratiques performatives d'essence éphémère qui s'inspirent ou s'appuient sur les supports de l'espace de la ville $»^{4}$. Néanmoins, les difficultés inhérentes aux tentatives de délimitation de la notion ont été maintes fois soulignées (Genin, 2013). Tout en en reconnaissant les limites, McAuliffe (2012) propose une distinction entre graffiti et street art. Le premier recouvre des pratiques artistiques centrées sur la production de lettrages et constitue une véritable sous-culture caractérisée par des codes propres et par son caractère illégal. Le second renvoie à une palette plus diversifiée de pratiques dont les œuvres sont destinées à un public plus large et sont, contrairement au graffiti, autorisées voire valorisées par les acteurs institutionnels et par le marché de l'art. Nombre de travaux soulignent néanmoins le caractère arbitraire et variable de ces catégorisations qui renvoient moins aux caractéristiques des œuvres qu'elles prétendent définir qu'au regard que l'on porte sur elles et à la légitimité qu'on leur accorde. Christophe Genin (2015) montre ainsi la diversité des critères d'évaluation sur lesquels reposent les tentatives de délimitation du street art, qu'ils soient de nature juridique, spatiale, esthétique ou encore morale. Selon l'approche retenue, le graffiti peut ou non y être inclus. Genin propose d'aborder la notion de street art à l'aune des processus de reconnaissance économique, sociale, esthétique et politique dont les œuvres font l'objet. L'on distingue alors les productions auxquelles les acteurs institutionnels et économiques confèrent une valeur artistique et marchande de celles qui ne bénéficient pas d'une telle reconnaissance, lesquelles recouvrent souvent des formes illégales. Tout en reconnaissant qu'il est difficile de proposer une définition définitive du street art tant son contenu est constamment renégocié, Ulrich Blanché (2015) y associe les caractéristiques suivantes: le street art relèverait d'un processus de création "auto-autorisé » (p.5), in situ, performatif et participatif. Par son mode de communication qui repose essentiellement sur l'image, il se distinguerait du graffiti caractérisé par la prépondérance du texte. Tout en gardant à l'esprit cette diversité des approches, nous choisissons ici d'utiliser alternativement " graffiti » et «street art » selon que nous renvoyons à la manière dont le créateur des œuvres analysées ici définit sa pratique ${ }^{5}$ ou à une compréhension plus englobante du street art en termes de pratiques artistiques, qui inclut le graffiti sans s'y limiter.

4 À partir du cas de Saint-Nazaire et de l'analyse du projet « Les Oides » né de l'initiative d'un graffeur anonyme, nous interrogeons les enjeux spatiaux de la valorisation urbaine du graffiti et du street art selon une double perspective. Il s'agit tout autant de préciser leur rôle dans la valorisation d'un espace urbain que de comprendre les logiques de la légitimation institutionnelle des pratiques artistiques au sein de l'espace urbain. Le projet de street art peut-il contribuer à la valorisation de l'espace dans lequel il s'inscrit? Quelle est sa capacité à influer sur le rapport à l'espace des usagers de la ville? Quels sont les acteurs qui interviennent dans sa valorisation urbaine et sur quels critères fondent-ils leur action? Nous envisageons en premier lieu le street art comme un facteur potentiel d'évolution des représentations et des pratiques associées aux 
espaces urbains dans lesquels il s'inscrit, et ce en dehors de tout dispositif de valorisation institutionnelle. Nous analysons ensuite les logiques qui président à sa légitimation dans l'espace de la ville, afin de comprendre ce qui fait ici obstacle à la valorisation par les acteurs publics d'un projet pourtant consensuel. À partir de ces réflexions, nous mettons en évidence l'écart entre la capacité effective de la production artistique à valoriser un territoire et les choix auxquels répond la décision politique de reconnaître ou non ce potentiel sous la forme d'une institutionnalisation.

5 Pour ce faire, cet article s'appuie sur une étude de terrain menée entre novembre 2017 et janvier 2018. La méthodologie mobilisée associe des analyses quantitatives et qualitatives et a pour ambition d'aborder notre objet à la fois du point de vue de la production (artiste), de la réception (public et commerçants locaux) et de l'institution (responsables municipaux). Le volet quantitatif repose sur l'analyse de 160 réponses à un questionnaire diffusé sur les pages Facebook et Instagram consacrées au projet des Oides et gérées par l'artiste ${ }^{6}$. Du fait de son mode de diffusion, ce questionnaire s'adressait explicitement à un public déjà familier du projet. L'objectif n'était pas d'évaluer la popularité des Oides auprès de la population locale dans son ensemble mais bien d'en cerner la réception par un public déjà informé, et notamment d'en comprendre les effets sur les représentations et les pratiques de la ville. Le nombre de répondants diffère selon les questions, l'échantillon de référence est donc variable et nous en précisons la taille lorsque cela est nécessaire. Le volet qualitatif repose quant à lui sur l'analyse de cinq entretiens semi-directifs réalisés avec le créateur anonyme des Oides $^{7}$, l'adjoint au maire de Saint-Nazaire en charge de la culture, le directeur de SaintNazaire Agglomération Tourisme, le co-gérant d'une boutique associative du centreville nazairien et un résident de Saint-Nazaire particulièrement impliqué dans le projet des Oides.

\section{Graffiti, street art et espace urbain : contexte et ancrage du projet artistique dans la ville}

\section{Saint-Nazaire, une ville portuaire en mutation}

6 Située sur l'estuaire de la Loire, Saint-Nazaire, quatrième port de commerce français et ville réputée pour ses chantiers navals, se développe à partir du milieu du XIX ${ }^{\mathrm{e}}$ siècle grâce à l'essor de son port et de l'industrie navale. La ville connaît des agrandissements successifs de ses installations portuaires ainsi que l'implantation de l'industrie aéronautique avant que la Seconde Guerre Mondiale ne marque un tournant majeur dans sa trajectoire. Détruite à $85 \%$ par les bombardements alliés, Saint-Nazaire est reconstruite selon un schéma fonctionnel qui entérine la séparation entre la ville et son port, par ailleurs coupé du tissu urbain par la monumentale base sous-marine léguée par l'armée allemande. D'abord considérés comme un exemple réussi de modernité urbanistique, les choix ayant présidé à la reconstruction sont remis en cause à partir des années 1970. Saint-Nazaire connaît dès lors les difficultés souvent associées aux villes reconstruites (déqualification du centre-ville, inadaptation du bâti reconstruit), à l'instar de Lorient et du Havre, auxquelles s'ajoutent bientôt les fluctuations de l'industrie navale (Desse, 1993 ; Gravari-Barbas, 2002).

7 Face à une situation de crise urbaine et sociale, la municipalité socialiste menée depuis 1983 par Joël Batteux se lance dans la restructuration d'un centre-ville alors en perte de 
vitesse (Desse, 1993), qui reste aujourd'hui encore fragile comme l'atteste la présence de nombreuses friches commerciales. À partir du milieu des années 1990, le projet "Ville-port» doit quant à lui réconcilier la ville avec son espace portuaire. La réhabilitation de la base sous-marine et l'implantation de nouveaux équipements urbains à proximité immédiate en sont les éléments centraux. Dans ce contexte de revalorisation de l'identité portuaire de Saint-Nazaire, les acteurs institutionnels développent par ailleurs une offre touristique essentiellement consacrée au patrimoine industriel et portuaire de la ville (Gravari-Barbas, 2002), regroupée depuis 2015 sous la bannière « Le Port de tous les Voyages ».

8 L'art et sa valorisation urbaine semblent contribuer à cette reconquête. La biennale d'art contemporain Estuaire a ainsi laissé plusieurs œuvres pérennes dans l'espace portuaire depuis 2007, à l'image de la Suite de triangles de Felice Varini et du Jardin du Tiers-Paysage de Gilles Clément, tandis que le street art a lui aussi récemment investi les alentours des bassins portuaires ${ }^{8}$. À plus petite échelle, la production artistique et sa valorisation occupent une place singulière dans la fabrique territoriale de la métropole Nantes-Saint-Nazaire. En favorisant les synergies entre le tourisme, l'art contemporain et le patrimoine, Estuaire contribue à la structuration métropolitaine en ce qu'elle favorise l'émergence d'une identité géographique cohérente pour un territoire estuarien jusqu'alors fragmenté (Coëffé et Morice, 2013).

Le contexte géographique nazairien est donc celui d'une ville moyenne de la façade atlantique fortement marquée par son identité portuaire et industrielle, et dont l'image peu attractive commence à évoluer sous l'effet des projets urbains menés depuis les années 1990. Le développement récent du street art semble alors constituer à la fois un indicateur et un levier potentiel de revalorisation urbaine. C'est dans ce contexte qu'un artiste anonyme lance en 2015 son projet « Les Oides », dont l'une des spécificités réside dans l'étroite relation qu'entretiennent les œuvres avec l'espace urbain nazairien.

\section{Le projet des Oides : une « chasse » aux œuvres entre promotion de la pratique artistique et mise en valeur du territoire nazairien}

10 Au cours de l'été 2015 , les Oides 9 , petits personnages bleus au graphisme simple et peints à la bombe, commencent à essaimer dans la région nazairienne (illustrations 1 et 2). Leur créateur est un graffeur amateur sans formation artistique revenu s'installer à Saint-Nazaire après avoir grandi en région parisienne. Son projet consiste en la réalisation d'une série qui s'enrichit chaque jour d'une nouvelle œuvre mettant en scène un ou plusieurs personnages dans une situation inédite. Selon le principe de la chasse au trésor, chaque création est photographiée et postée sur les réseaux sociaux pour servir d'indice aux amateurs désireux de la trouver. Relayé par la presse locale, le projet rencontre rapidement un écho favorable au sein de la population nazairienne, donnant lieu à la structuration progressive d'une petite communauté virtuelle regroupant des amateurs plus ou moins assidus de ce qui relève désormais d'une « chasse aux Oides» (voir la $2^{\mathrm{e}}$ partie de l'article sur le profil du public concerné). La page Facebook ${ }^{10}$ du projet compte ainsi 1752 mentions «J'aime ", tandis que le compte Instagram compte 897 abonnés $^{11}$. Outre ses réalisations quotidiennes, l'artiste propose ponctuellement des « chasses » dans un espace-temps limité et des expositions dans des galeries locales. À l'été 2017 , l'apparition du $500^{\mathrm{e}}$ personnage marque la fin du projet dans sa forme originelle, l'artiste se concentrant désormais sur des réalisations plus 
travaillées et moins régulières. Notons que les Oides relèvent d'une initiative personnelle mise en œuvre sans autorisation de la municipalité : juridiquement, il s'agit d'une forme de vandalisme. C'est donc d'un projet sans légitimité institutionnelle que nous proposons ici l'analyse géographique.

Illustration 1 - Exemple de Oides, \#117

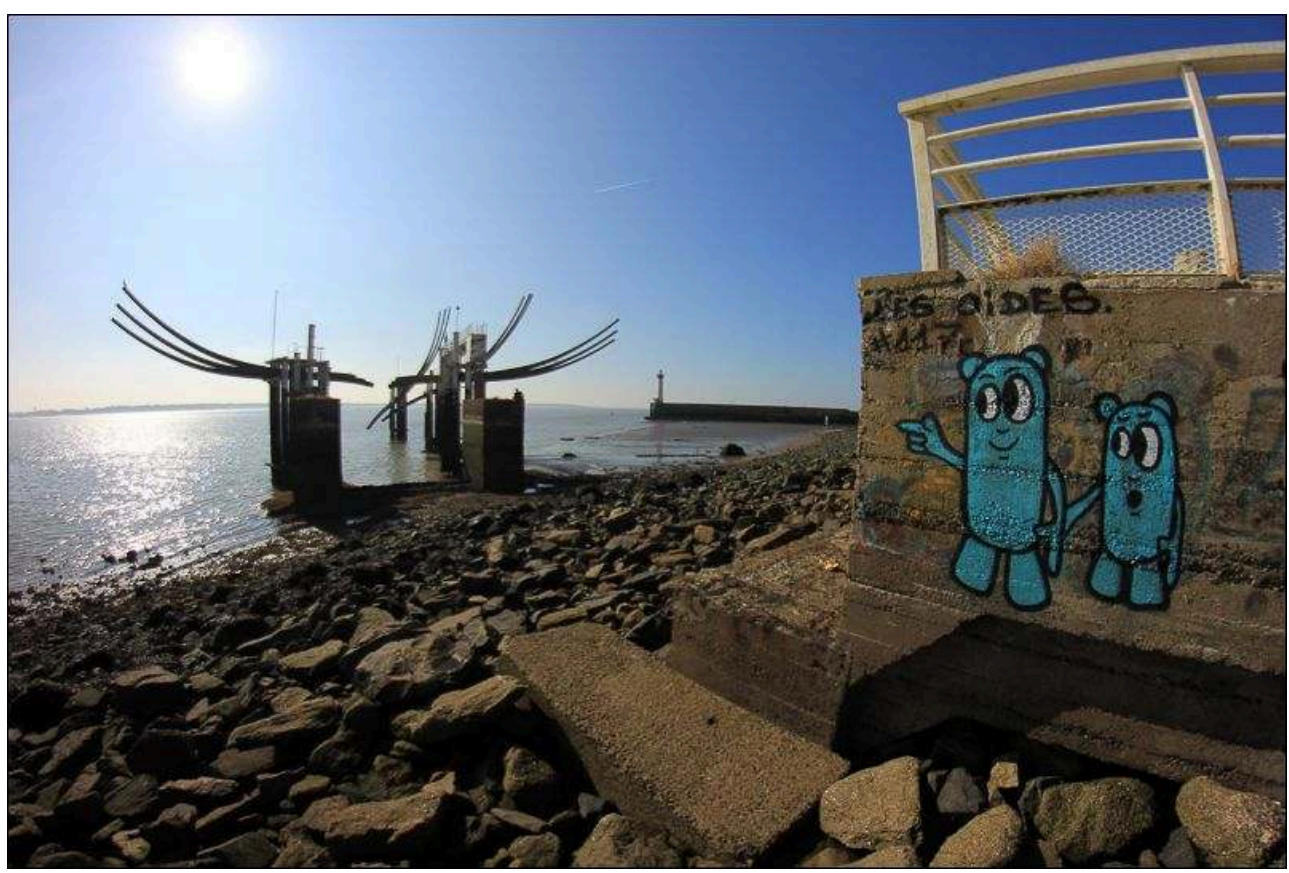

Source : photographie de l'artiste, 2016.

Illustration 2 - Exemple de Oides, \#129

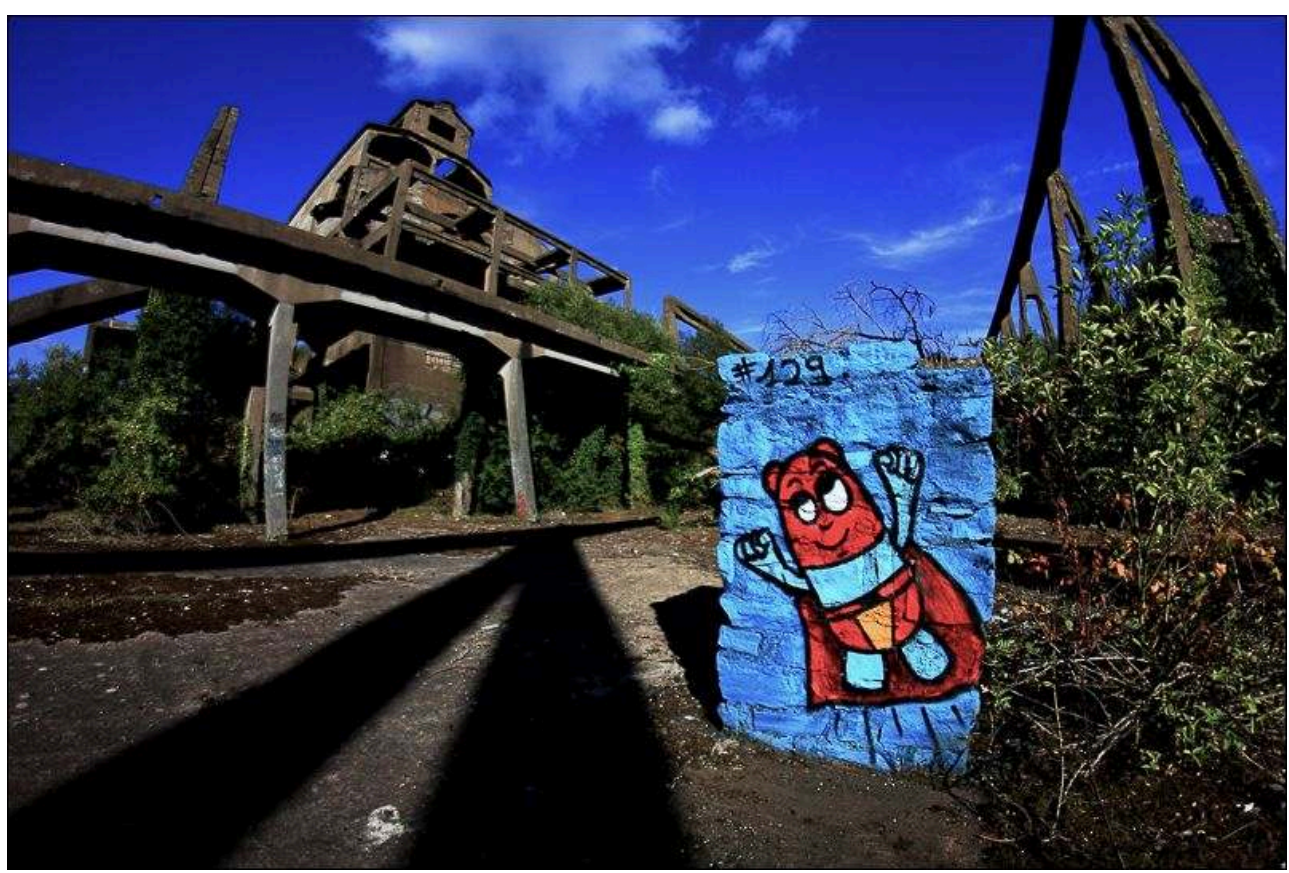

Source : photographie de l'artiste, 2016. 
11 L'invasion de l'espace nazairien par les œuvres fait partie intégrante du projet. À l'exception de quelques personnages, l'artiste se concentre essentiellement sur un périmètre compris entre Lorient, Nantes et Saint-Nazaire, cette dernière abritant la majorité des créations (illustration 3). À l'échelle de l'agglomération nazairienne (illustration 4), les Oides sont particulièrement présents le long de la côte, espace de promenade apprécié de la population locale, ainsi que sur les pourtours des bassins portuaires et dans les espaces de friches, à l'image des anciennes forges de la commune limitrophe de Trignac (illustration 2). En termes de supports, l'artiste tire parti du bâti urbain (palissades, bâtiments abandonnés, murs de bâtiments non habités) comme des éléments naturels (rochers). Cette dispersion des personnages au sein de l'espace urbain appelle de la part du public une démarche active de recherche et donc une pratique de l'espace orientée par la répartition des œuvres dans la ville.

Illustration 3 - Quand l'artiste cartographie sa production à l'échelle nationale...

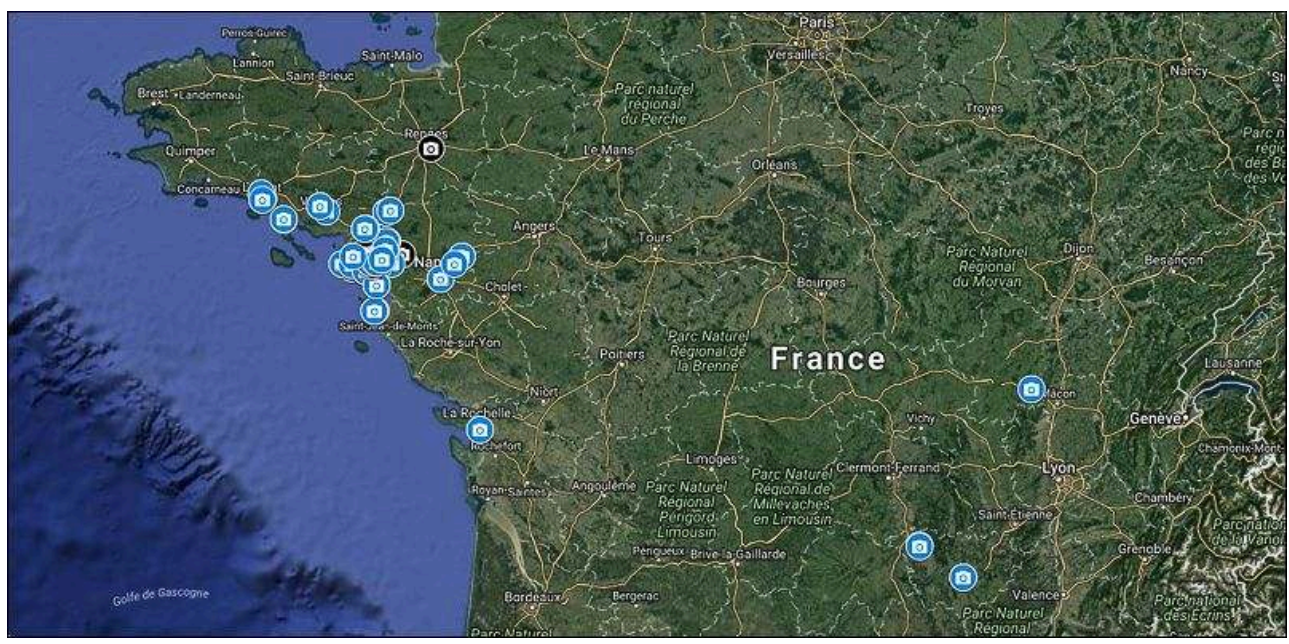

Source : extrait de la carte en ligne « Les Oides », https://bit.ly/2xAPSb9, consultée le 2 juin 2018.

Illustration 4 - ... et à l'échelle de l'agglomération nazairienne

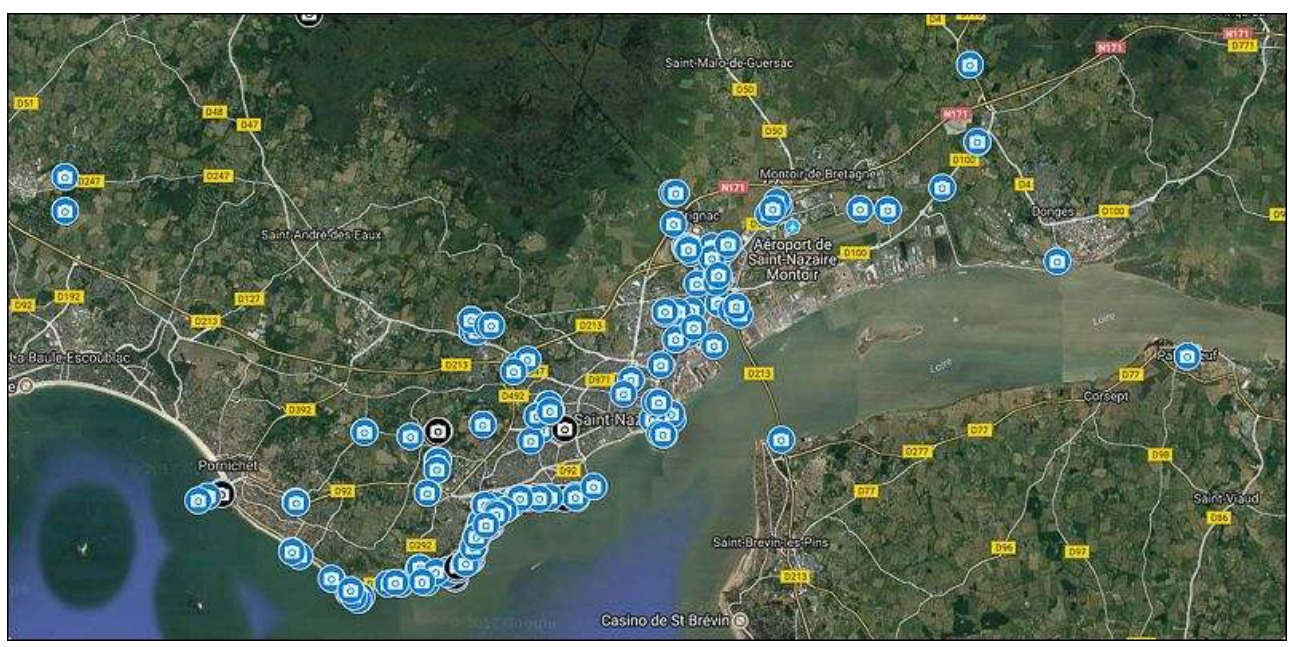

Source : extrait de la carte en ligne « Les Oides », https://bit.ly/2xAPSb9, consultée le 2 juin 2018. 
12 L'objectif revendiqué par l'artiste est double : «ouvrir [un large public] au milieu du graff' et faire une publicité pour Saint-Nazaire en même temps » (entretien, novembre 2017). Il s'agit en premier lieu de contribuer à un changement de regard sur la pratique du graffiti elle-même, à laquelle l'artiste cherche à sensibiliser un public très large. Cela se traduit d'une part par une absence volontaire de politisation du message véhiculé par les Oides, dont les mises en scène relèvent d'une iconographie bon enfant et consensuelle. D'autre part, l'attention particulière prêtée au choix des lieux dans lesquels elles sont peintes allie recherche d'originalité et souci d'accessibilité : l'artiste retient essentiellement des emplacements dont l'accès ne présente aucun danger. Il tient par ailleurs à jour une cartographie en ligne ${ }^{12}$ indiquant l'emplacement de la plupart des personnages, à l'exception des quelques œuvres situées dans des lieux dont la pratique présente des risques. Cette démarche relève là encore d'une volonté de rendre le projet accessible non seulement à un public averti, mais aussi à un public moins prompt à trouver par lui-même l'emplacement des personnages. On s'éloigne dès lors des origines subversives et contestataires du graffiti pour en favoriser une expression plus consensuelle, qui tend en termes de contenu et de public visé vers les caractéristiques du street art institutionnalisé. Dans cette perspective, l'espace urbain est envisagé comme un support matériel au service de la valorisation de la pratique artistique et constitue une ressource dont le graffiti tire parti, notamment grâce à la visibilité qu'il offre.

Le projet a également pour ambition de mettre en valeur Saint-Nazaire et son patrimoine par la pratique du graffiti. Il s'agit ainsi d'« emmener les gens dans des endroits où ils ne seraient jamais allés autrement» (entretien, novembre 2017), à l'image des friches urbaines ou des blockhaus. Par les emplacements choisis, la recherche des Oides est censée susciter la découverte de sites jugés intéressants par l'artiste mais peu visibles dans le cadre des pratiques quotidiennes de l'espace urbain. Dans cette optique, l'espace devient enjeu : il ne s'agit plus de le considérer comme un support permettant de valoriser la pratique artistique qui s'y inscrit, mais bien d'utiliser le graffiti comme outil de mise en valeur de l'espace urbain.

14 Le graffeur déploie ainsi une stratégie qui repose à la fois sur le contenu de ses œuvres et sur leur localisation pour atteindre un double objectif: valoriser la pratique artistique tout comme l'espace urbain dans lequel elle s'inscrit, lequel constitue à la fois une ressource et un enjeu, un moyen et un but. Nous analysons à présent la réception effective du projet afin de déterminer si les objectifs affichés correspondent ou non à la manière dont le public s'en saisit. Il s'agit de comprendre si ce projet artistique peut, hors de tout dispositif institutionnel de valorisation, influencer la manière dont les usagers de la ville la perçoivent et la pratiquent.

\section{Représentations et pratiques de la ville : les Oides, un facteur d'évolution des territorialités locales?}

\section{Un projet grand public à la portée essentiellement locale}

Les 160 réponses au questionnaire permettent de préciser le profil du public touché par le projet. $67 \%$ des répondants sont des femmes, $33 \%$ des hommes. Si la moitié d'entre eux ont entre 25 et 44 ans, les autres catégories sont relativement bien représentées, à l'exception des plus de 65 ans dont la part est plus modeste (illustration 5). Il s'agit 
donc d'un public varié en termes d'âge. La dimension " grand public » revendiquée par l'artiste est également confirmée par l'importance du public familial. Sur les 78 répondants qui précisent leurs habitudes de recherche des Oides, 34 personnes (soit $44 \%)$ déclarent ainsi chercher les personnages en famille et avec leurs enfants.

Illustration 5 - Répartition des répondants au questionnaire par catégories d'âge

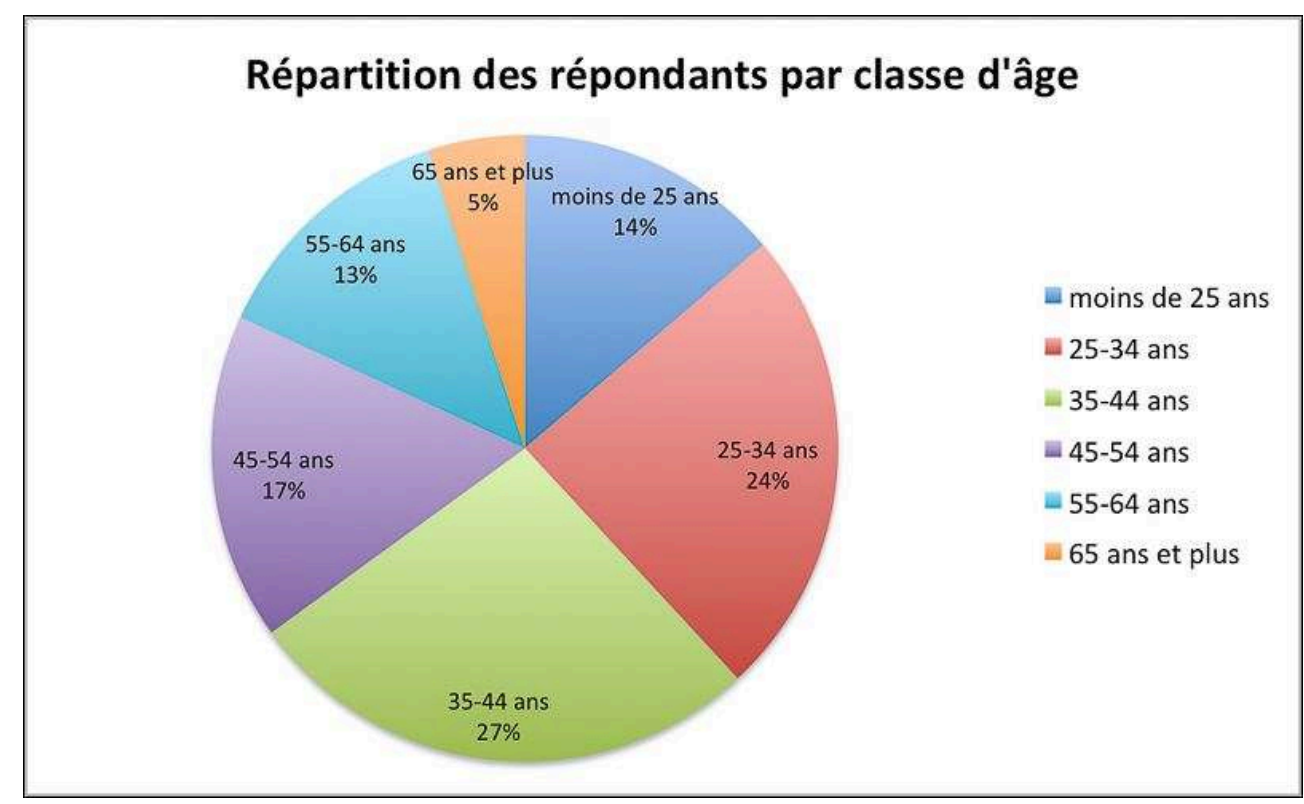

Auteur: A. Le Gallou, 2018.

Ce public se montre inégalement impliqué dans la recherche des personnages : $34 \%$ des répondants déclarent chercher activement les œuvres, les autres se contentant de découvertes occasionnelles au hasard de leurs déplacements. Par ailleurs, seules $21 \%$ des personnes interrogées s'étaient rendues à l'une des expositions et $6 \%$ avaient participé aux "Chasses aux Oides» organisées par l'artiste. Parmi ces quelques amateurs particulièrement assidus, un résident de Saint-Nazaire évoque la création d'un groupe de recherche d'une demi-douzaine de personnes et souligne la capacité du projet à susciter des rencontres fondées sur un intérêt commun pour les Oides. Au-delà de ce noyau formé de personnes extrêmement impliquées, dont on peut évaluer le nombre à quelques dizaines, on a donc affaire à un public intéressé sans être particulièrement assidu. Les pratiques engendrées par les œuvres couvrent ainsi un large spectre allant d'une recherche systématique à un intérêt passif.

Par ailleurs, la répartition des répondants par lieu de résidence montre que le public touché est local (illustration 6). Plus de la moitié d'entre eux résident à Saint-Nazaire (63\% dans la communauté d'agglomération nazairienne), tandis que seuls $11 \%$ habitent hors du département. $85 \%$ des personnes interrogées (résidents inclus) déclarent en outre se rendre à Saint-Nazaire au moins une fois par mois. Conformément aux ambitions affichées par l'artiste, le projet touche donc un public relativement large en termes de profil mais essentiellement local, très majoritairement constitué d'habitants et d'usagers fréquents. Cela s'explique aisément par la concentration des œuvres dans la région nazairienne, mais il est aussi possible d'y voir l'effet de l'absence de valorisation institutionnelle, notamment touristique, du projet. 
Illustration 6 - Répartition des répondants au questionnaire par lieu de résidence

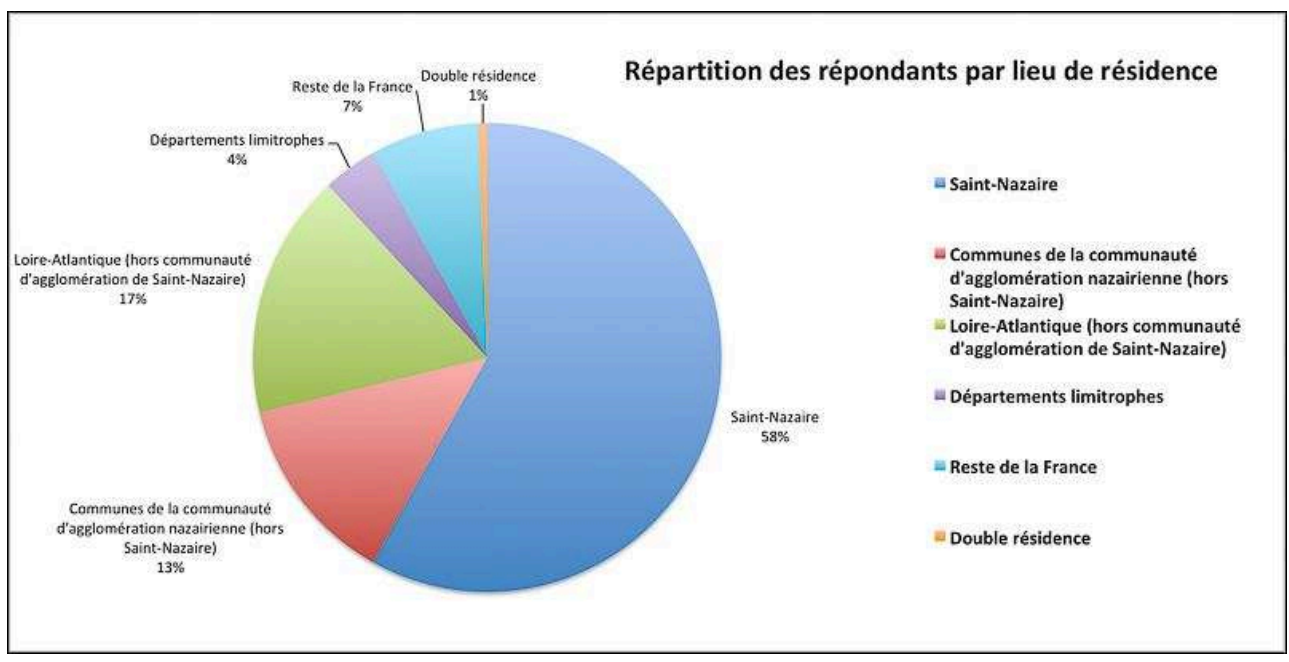

Auteur: A. Le Gallou, 2018.

\section{Graffiti, représentations et pratiques spatiales}

Les représentations et pratiques spatiales suscitées par les Oides auprès du public local montrent que le graffiti peut influencer l'appropriation de l'espace nazairien par ses usagers. La présence de ces petits personnages fait d'abord évoluer les représentations de la ville : $43 \%$ des répondants considèrent ainsi que les Oides ont changé l'image qu'ils avaient de Saint-Nazaire. Selon eux ${ }^{13}$, le projet confère à la ville une image dynamique ( $16 \%$ des répondants) et une identité artistique (17\%), mais il permet surtout de la rendre moins triste $(28 \%)$. Selon l'un des répondants, les Oides apportent " une drôle d'animation qui change de la morosité de la ville ", tandis qu'un autre salue leur " légèreté et le nouveau regard » qu'ils contribuent à faire naître sur une ville à l' " image triste, grise et moche ». Sont également évoqués le caractère ludique et original du projet, sa dimension conviviale et sa capacité à valoriser le patrimoine local. La prolifération des personnages dans l'espace urbain nazairien incite donc habitants et usagers à reconsidérer une vision de Saint-Nazaire souvent négative. Peut-être est-ce d'autant plus vrai que l'artiste met parfois en scène les marqueurs spatiaux et l'identité portuaire de la ville. C'est par exemple le cas du \#417 de la série, réalisé aux abords du port et qui représente le pont de Saint-Nazaire (illustration 7). On peut supposer que la mobilisation de référents spatiaux partagés accentue la revalorisation de la ville aux yeux des habitants en suscitant une forme de fierté locale: Saint-Nazaire devient non seulement le support mais aussi le sujet du projet des Oides, confirmant ainsi l'intérêt artistique dont la commune peut faire l'objet. 


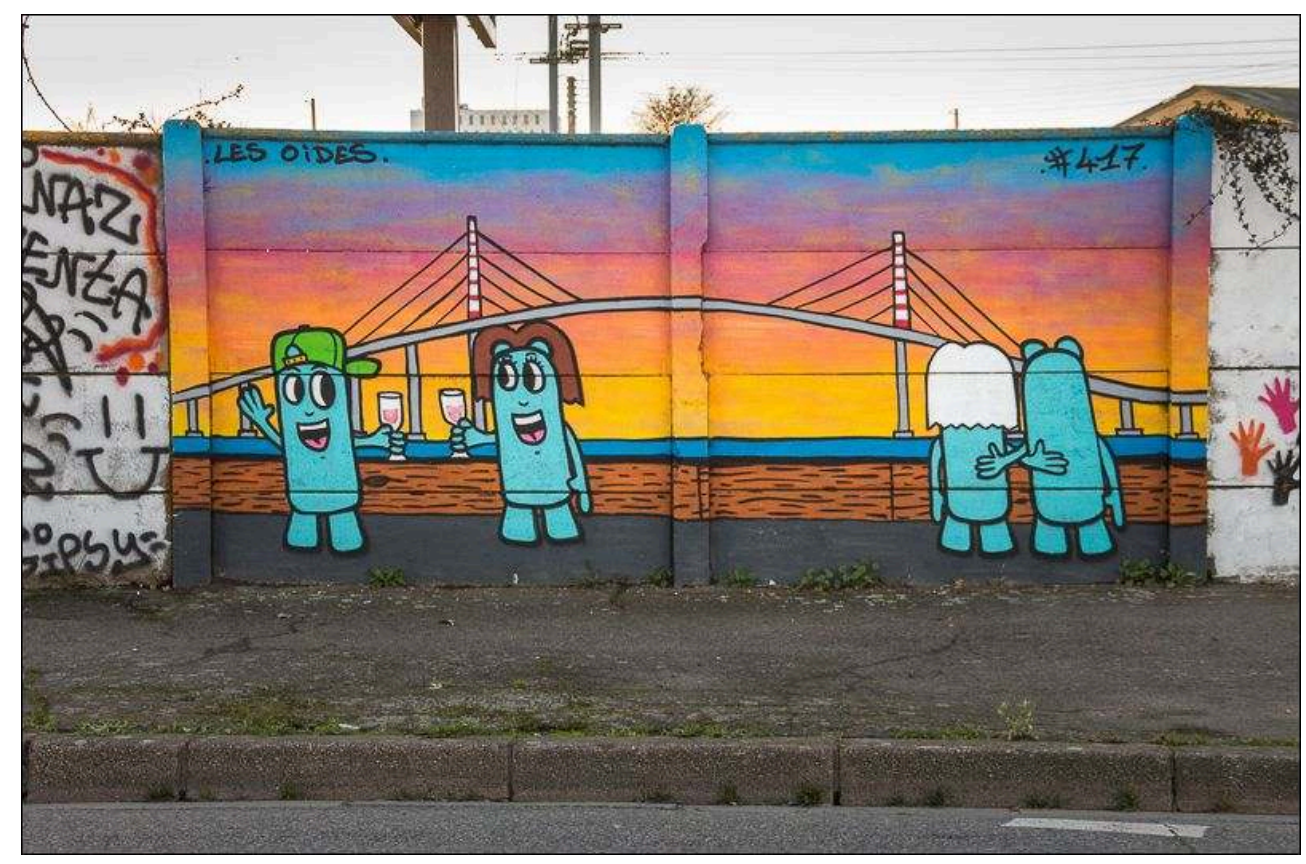

Auteur: A. Le Gallou, 2017.

Les Oides contribuent également à une évolution des pratiques de l'espace urbain nazairien. Si cela concerne une part minoritaire de répondants (seuls $23 \%$ d'entre eux déclarent une influence du projet sur leurs pratiques), les réponses ${ }^{14}$ permettent de faire ressortir deux constats. Premièrement, 11 des 31 personnes ayant précisé la nature de l'influence des Oides sur leurs pratiques indiquent que le projet les a incitées à se promener davantage à Saint-Nazaire. Il influe donc sur l'intensité et la fréquence de l'appropriation de l'espace proche dans le cadre de pratiques récréatives. Deuxièmement, les Oides suscitent la découverte et la pratique de nouveaux espaces au sein de la ville. $41 \%$ de l'ensemble des répondants déclarent ainsi que la recherche des personnages les a amenés à se rendre dans des lieux qu'ils ne fréquentaient pas habituellement. Parmi eux sont cités des types d'espaces génériques (bois, champs, chemins) et des lieux précisément identifiés. Trois espaces ou types de lieux sont évoqués de manière récurrente: la côte nazairienne, les lieux abandonnés et les blockhaus, encore nombreux dans la région nazairienne. Le projet contribue donc à éveiller un intérêt pour les marges urbaines que constituent les friches, auparavant situées hors du champ des pratiques quotidiennes et désormais davantage intégrées à l'espace vécu d'une partie des habitants. Cela répond au souhait de l'artiste d'« emmener les gens dans des endroits où ils ne seraient jamais allés autrement » (entretien, novembre 2017).

20 En dépit de sa bonne réception globale par le public nazairien, le projet suscite quelques critiques. $6 \%$ des répondants déclarent ainsi ne pas l'apprécier, soulignant le caractère invasif des personnages, le manque de renouvellement du concept et une faible qualité esthétique. Là où la dissémination des œuvres représente pour beaucoup une manière d'égayer l'espace urbain, elle constitue pour d'autres une forme de pollution visuelle. Le projet soulève également des questions relatives aux conceptions divergentes de la mise en valeur du patrimoine local. L'artiste mentionne ainsi un désaccord avec une association de protection des blockhaus de la Seconde Guerre 
Mondiale, pour qui l'utilisation de ces bâtiments comme supports artistiques relève de la dégradation, tandis que l'artiste conçoit sa démarche comme une forme de valorisation susceptible d'attirer un nouveau public. Cette tension ouvre des pistes de recherches quant au potentiel $\mathrm{du}$ street art comme outil de revalorisation du patrimoine.

Qu'il s'agisse des représentations associées à la ville ou des pratiques dont elle fait l'objet, on constate donc un réel impact du projet des Oides sur la manière dont les usagers s'approprient l'espace urbain nazairien. L'analyse invite ainsi à s'intéresser non plus à l'espace de la pratique artistique mais aux territoires vécus par son public, territoires reconfigurés par l'inscription du street art dans la ville. Celle-ci favorise à la fois une revalorisation de l'image de Saint-Nazaire et une extension des espaces connus et pratiqués. Dès lors se pose la question des enjeux d'une éventuelle valorisation institutionnelle alors que la capacité du projet à susciter de nouvelles formes de découverte de la ville semble établie. Cette hypothétique valorisation se heurte pourtant à un certain nombre de difficultés qui mettent en évidence le décalage entre l'intérêt du projet et les arguments qui président à sa valorisation. Pourquoi, alors que le projet est bien accepté et correspond dans ses contenus et dans ses ambitions aux orientations d'un street art institutionnalisé, les acteurs publics ne se saisissent-ils pas de cette initiative locale?

\section{D'une acceptation de fait aux logiques de légitimation de l'art en ville : une difficile valorisation institutionnelle}

\section{Trajectoire de professionnalisation et évolution de l'inscription spatiale du projet}

De plus en plus connu et accepté, le projet des Oides change de forme et de mode d'inscription spatiale sous l'effet conjoint d'une bonne réception de la part du public, des choix professionnels de l'artiste et de l'intervention de nouveaux acteurs. L'acceptation progressive du projet modifie les modalités de production de l'artiste, qui décrit ainsi l'évolution constatée en deux ans: «au début ça a été vraiment du vandalisme, dans le sens où je sortais la nuit pour aller faire les personnages, en étant caché, et aujourd'hui je ne vais pas dire que c'est légal mais c'est mieux vu, si je peins dans des endroits où il y a un peu de passage en pleine journée, les gens vont s'arrêter, vont venir discuter parce qu'ils ont déjà vu le personnage [...] donc aujourd'hui c'est mieux perçu qu'à l'époque » (entretien, novembre 2017). Malgré son caractère illégal, le projet bénéficie donc de la sympathie du public local comme de la tolérance de la municipalité, ce qui facilite son implantation dans l'espace urbain.

L'arrêt de la série de 500 personnages à l'été 2017 au profit d'œuvres plus travaillées mais moins régulières répond par ailleurs à une volonté d'évolution professionnelle de la part de l'artiste, désireux de s'affranchir du rythme soutenu que lui imposait la réalisation quotidienne d'un nouveau personnage mais aussi de pouvoir à terme vivre de sa pratique artistique. L'intervention progressive de nouveaux acteurs sous la forme de commandes répond à cette ambition. Plusieurs structures privées ont ainsi sollicité le graffeur pour la réalisation d'œuvres sur leurs murs, à l'image entre autres de l'école 
Saint-Jean-Sainte-Bernadette et de commerçants du centre-ville de Saint-Nazaire. La boutique associative «Pas Que Beau » est par exemple à l'origine de la fresque dite du passage des Halles au cœur du centre-ville (illustration 8). Réalisée sur un mur appartenant à la boutique et financée conjointement par six commerçants du quartier, elle répond à une volonté de ces derniers de diversifier les fonctions du centre-ville en proposant une offre culturelle qui permette de valoriser les artistes locaux. L'intérêt économique n'est pas absent de l'initiative, certains commerçants espérant qu'une dynamisation par l'art d'un centre-ville fragilisé profite également à la fréquentation des commerces.

Outre l'étape que cela représente dans la trajectoire professionnelle de l'artiste, ces éléments suscitent une évolution des modalités d'inscription spatiale du projet dans la ville. Les supports proposés permettent au graffeur de s'exprimer dans des espaces centraux à la visibilité importante. Dès le lancement du projet, l'artiste a fait le choix de ne pas peindre ses personnages sur des murs habités, d'où une présence limitée dans le centre-ville de Saint-Nazaire. Il n'accède à une forme de centralité que grâce à l'acceptation sociale de ses œuvres, la trajectoire professionnelle permettant une trajectoire spatiale de la périphérie vers le centre.

Illustration 8 - La fresque du Passage des Halles

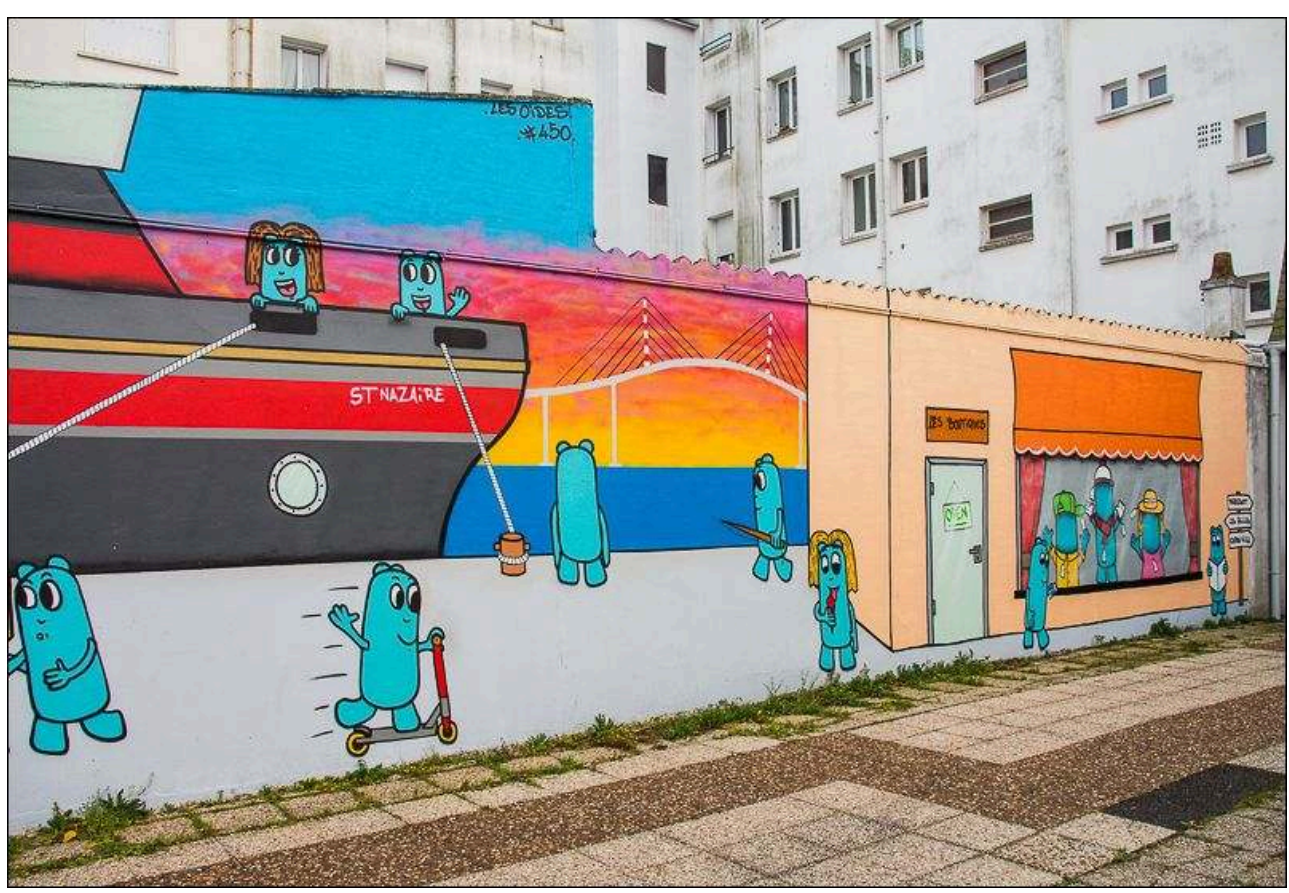

Réalisée à l'été 2017, elle est à l'initiative d'un regroupement de commerçants du centre-ville de SaintNazaire.

Auteur : A. Le Gallou 2017.

Cette évolution du projet et de son inscription dans l'espace urbain nazairien traduit un intérêt croissant pour les Oides de la part du public local comme des acteurs privés évoqués plus haut. Cette progressive acceptation aboutit à une forme de valorisation non officielle et non concertée qui offre une visibilité croissante au projet et pourrait constituer un préalable à une éventuelle valorisation institutionnelle du projet par les pouvoirs publics. Dans le contexte actuel d'une réflexion sur la présence de l'art dans l'espace public nazairien, cela se heurte pourtant à certaines difficultés. 


\section{Une difficile valorisation institutionnelle des Oides}

26 support de valorisation ludique de Saint-Nazaire semble établie, la valorisation institutionnelle du projet ne va pas de soi. L'artiste s'y montre pourtant favorable et initie lui-même un dialogue avec la municipalité. En s'inspirant des réflexions sur la construction du graffiti (envisagé dans un sens large) comme objet d'action publique à l'échelle municipale (Vaslin, 2017), il est alors intéressant d'analyser les raisons de cette difficile institutionnalisation.

Perçu comme une nuisance à l'ordre esthétique urbain ou légitimé en tant qu'objet culturel selon les périodes et les contextes (micro-)locaux, le graffiti peut faire l'objet d'une série de dispositifs de traitement allant de la répression à la valorisation (Vaslin, 2017). À Saint-Nazaire, le cas des Oides illustre selon l'adjoint au maire de Saint-Nazaire en charge de la culture la difficile position des acteurs publics vis-à-vis d'œuvres bien acceptées par la population mais qui relèvent juridiquement du vandalisme. Le projet bénéficie d'une tolérance de la part de la municipalité, qui choisit de ne pas s'en tenir à un effacement systématique des personnages: "les Oides sont nés d'une initiative artistique purement privée sans autorisation préalable de la ville. Parce que le message n'est pas subversif, parce que c'est bon enfant, on tolère. Mais imaginons qu'un autre graffeur développe des messages plus subversifs sur les mêmes lieux que les Oides, qui entrent moins en écho avec la population, que fait la mairie? Doit-elle effacer l'un et pas l'autre, alors que les deux œuvres sont de même initiative et ne sont pas légales? [...] Il y a une forme de bienveillance, mais ce serait très différent si le message était plus anguleux" (entretien, janvier 2018). Le contexte de production des œuvres apparaît donc déterminant: l'acceptation de fait ne permet pas d'aboutir à une institutionnalisation a posteriori d'un projet né dans l'illégalité, car cela nécessiterait la délicate définition de critères permettant de distinguer des initiatives juridiquement identiques.

Les arguments avancés en matière de valorisation touristique sont similaires. Le directeur de Saint-Nazaire Agglomération Tourisme souligne la nécessaire concertation dont les Oides devraient faire l'objet pour prétendre à une valorisation touristique : « il faudrait réfléchir aux éléments que l'on veut mettre en valeur dans le paysage urbain [...] quel message transmet-on? Au-delà de l'œuvre, on veut mettre en valeur l'environnement dans lequel elle s'insère, il y a nécessairement un message autour du patrimoine bâti, culturel, historique, naturel » (entretien, janvier 2018). Une éventuelle valorisation ne prendrait donc pas la forme d'une promotion des œuvres existantes, mais nécessiterait de prévoir la réalisation de nouvelles œuvres selon des objectifs et dans des espaces identifiés en accord avec les acteurs publics. Par ailleurs, le projet dans sa configuration actuelle se heurte à des impératifs de régulation et de sécurité relatifs à la gestion des flux, à la protection d'espaces littoraux parfois fragiles et à l'engagement de la responsabilité légale de Saint-Nazaire Agglomération Tourisme en cas de promotion d'œuvres situées à des emplacements dangereux. Selon son directeur, «le développement touristique ne peut se faire que dans un cadre légal ». Ces freins ne se traduisent cependant pas par une totale indifférence au projet, puisque celui-ci est intégré à la liste des œuvres de street art que propose Saint-Nazaire Agglomération 
Tourisme sur son site internet, liste dans laquelle les Oides constituent d'ailleurs le seul projet ne relevant pas d'une logique de partenariat entre l'artiste et les acteurs publics.

$\mathrm{Ni}$ réprimé ni véritablement légitimé, le projet échappe ainsi à tout dispositif institutionnel d'encadrement. Légalité et concertation avec les acteurs publics apparaissent par ailleurs nécessaires à une valorisation institutionnelle du street art à Saint-Nazaire. Les arbitrages de ces acteurs répondent à des critères qui dépassent le seul potentiel du projet considéré, fût-il par ailleurs conforme aux caractéristiques d'une production artistique institutionnalisée en termes de contenu et d'objectifs. Cela explique le fait que les Oides, malgré leur potentiel en matière de valorisation de la ville, ne constituent pas une priorité dans une stratégie municipale actuellement en cours d'élaboration.

\section{Entre promotion des artistes locaux et ambitions internationales, une stratégie de valorisation du street art en gestation}

30 La démarche de concertation des «Rendez-Vous de la Culture » menée autour de la politique culturelle de Saint-Nazaire a abouti en juin 2017 au constat d'une offre satisfaisante mais néanmoins en retrait concernant la présence de l'art et notamment du street art dans l'espace public. La biennale « Estuaire » a pourtant montré la voie dès 2007 en matière d'art dans la ville. Cependant, ce n'est que depuis 2015 qu'une forme de street art soutenue par les institutions fait réellement son apparition dans l'espace public. Est alors initié le partenariat toujours en cours avec le festival de musique «Les Escales ", qui permet chaque année la réalisation de fresques sur les façades de l'office HLM de la ville par des artistes invités. Le festival des cultures urbaines "Bouge» soutenu par la ville permet également de développer quelques œuvres avec l'accord de la municipalité, tandis que les commémorations du centenaire du débarquement américain à Saint-Nazaire ont donné lieu en 2017 à la réalisation d'une fresque des Toqué Frères consacrée à la construction navale.

31 Comme c'est le cas à Paris (Vaslin, 2017), street art et graffiti s'affirment ainsi comme des objets de gouvernement urbain à Saint-Nazaire. Se pose alors la question des ambitions et des choix de la municipalité en la matière. Le cas du XIII ${ }^{e}$ arrondissement de Paris, où le street art constitue un élément majeur de la politique culturelle (Kullmann, 2015), constitue pour l'adjoint à la culture un exemple inspirant. Sensible au potentiel en matière de valorisation et d'attractivité urbaine, il souligne l'enjeu du choix entre deux modes de mise en valeur dans l'espace urbain. Le premier consiste à mettre à la disposition des graffeurs des espaces de libre expression destinés essentiellement aux artistes locaux, tandis que le second repose sur le principe de la commande publique. Les implications de l'une et l'autre possibilités en termes de contenu et de visibilité sont différentes: l'une implique de prendre le risque de voir apparaître des œuvres au contenu non consensuel tout en restant fidèle aux racines subversives du graffiti, l'autre valorise un street art institutionnalisé qui permet un relatif contrôle du contenu des œuvres. Cette dernière est par ailleurs plus susceptible d'attirer des artistes internationalement reconnus, dont les œuvres constitueraient un facteur d'attractivité potentiel tant pour un public extérieur que pour d'autres artistes. Définir une ligne politique en matière de valorisation urbaine du street art implique ainsi de se positionner par rapport à la tension intrinsèque entre la dimension potentiellement contestataire des pratiques qu'il recouvre et les nécessaires mutations 
qu'entraîne leur institutionnalisation. Si les deux modes de valorisation ne sont pas exclusifs l'un de l'autre, la détermination de leur poids respectif dans la stratégie municipale s'avère délicate.

La mise en place d'une dynamique institutionnelle de valorisation du street art à SaintNazaire n'en est qu'à ses débuts et ses modalités devraient être précisées au cours de l'année 2018. Force est cependant de constater que les œuvres actuellement valorisées correspondent clairement au modèle de la commande publique qui prévaut dans le $\mathrm{XIII}^{\mathrm{e}}$ arrondissement de Paris. On remarque d'ailleurs des convergences: l'artiste chilien Inti, qui a signé trois des fresques parisiennes, a également réalisé une œuvre à Saint-Nazaire en 2015. Si les moyens et les ambitions de la municipalité sont pour l'instant sans commune mesure avec ceux du XIII ${ }^{e}$ arrondissement, la ville offre néanmoins des supports susceptibles d'attirer des artistes renommés, à commencer par la monumentale base sous-marine. Ces possibilités esquissent une stratégie dans laquelle la place des initiatives spontanées reste à déterminer.

\section{Conclusion}

L'exemple des Oides offre ainsi à l'analyse un projet de graffiti illégal mais qui se rapproche d'un street art institutionnalisé par la nature consensuelle et grand public de son contenu et de ses objectifs. Indépendamment de toute promotion institutionnelle, ce projet suscite une évolution des représentations et des pratiques des usagers de la ville, auxquels il ouvre de nouvelles perspectives d'appropriation de l'espace urbain. Il constitue en ce sens un facteur de valorisation de la ville dans laquelle il s'inscrit, tout en contribuant à la reconfiguration des territorialités individuelles de ses habitants et usagers. Malgré ce constat, la légitimation institutionnelle des Oides se heurte à l'incompatibilité entre la nature d'une initiative illégale et non concertée d'une part et les orientations retenues par la municipalité d'autre part. Plus que le contenu et les ambitions $\mathrm{du}$ projet, en adéquation avec les exigences d'une valorisation institutionnelle, c'est l'impératif d'une production dans un cadre légal et négocié en amont qui prévaut. Les logiques institutionnelles de la valorisation du street art le maintiennent alors dans une situation intermédiaire où son acceptation de fait se substitue à une véritable reconnaissance. Dès lors, une promotion plus informelle des Oides est permise par la convergence d'initiatives privées : ce sont les acteurs privés (commerçants, établissements d'enseignement ou encore particuliers) et non la puissance publique qui assurent la valorisation urbaine du projet. Les Oides expriment en définitive un paradoxe qui touche à la conformité à la norme: alors même que le projet tend spontanément vers une forme de street art institutionnalisée, les modalités de la valorisation par les acteurs publics ne lui permettent pas de prétendre à une légitimation. En ce sens, l'exemple de Saint-Nazaire manifeste la complexité persistante des relations entre le street art et les espaces urbains dans lesquels il s'inscrit, dans un contexte où la pratique artistique est pourtant de plus en plus mobilisée au service de l'attractivité urbaine. 


\section{BIBLIOGRAPHY}

Blanché U., 2015. Qu'est-ce que le Street art ? Essai et discussion des définitions. Cahiers de Narratologie [En ligne], 29 | 2015, mis en ligne le 31 janvier 2016, consulté le 7 juillet 2018. URL : http://narratologie.revues.org/7397 ; DOI : 10.4000/narratologie.7397

Coëffé V., Morice J.-R., 2013. Patrimoine et création dans la fabrique territoriale : l'estuaire ligérien ou la construction d'un territoire métropolitain. Norois [En ligne], 228 | 2013, mis en ligne le 30 septembre 2015, consulté le 04 janvier 2018. URL : http://norois.revues.org/4746 ; DOI : $10.4000 /$ norois. 4746

Desse R.-P., 1993. Saint-Nazaire ou le centre retrouvé : un exemple d'urbanisme commercial novateur. Norois, $n^{\circ} 158$, p. 235-252. DOI: 10.3406/noroi.1993.6480

Genin C., 2013. Le street art au tournant, reconnaissance d'un genre. Bruxelles Les Impressions nouvelles, $272 \mathrm{p}$.

Genin C., 2015. Le street art : de nouveaux principes ? Cahiers de Narratologie [En ligne], 29 | 2015, mis en ligne le 08 janvier 2016, consulté le 03 juin 2018. URL : http://journals.openedition.org/ narratologie/7396

Gravari-Barbas M., 2002. Tourism policies in French post-2 ${ }^{\text {nd }}$-World-War reconstructed cities: Saint-Nazaire, Le Havre and Lorient. City Tourism 2002: Proceedings of European Cities Tourism's International Conference in Vienna, p. 251-264.

Grésillon B., 2002. Berlin, métropole culturelle. Paris, Belin, 351 p.

Grésillon B., 2014. Géographie de l'art. Ville et création artistique. Paris, Editions Economica, 254 p.

Guinard P., 2014. Johannesburg. L'art d'inventer une ville. Rennes, Presses Universitaires de Rennes, $326 \mathrm{p}$.

Iveson K., 2010. The wars on graffiti and the new military urbanism. City, n 14, p. 115-134.

Kullmann C., 2015. De l'exposition de la Tour Paris 13 au concept de musée à ciel ouvert. Téoros [En ligne], 34, 1-2 | 2015, mis en ligne le 25 avril 2016, consulté le 02 juin 2018. URL : http:// journals.openedition.org/teoros/2776

Kullmann C., 2017. La valorisation du projet urbain par la dimension artistique. Quelles perspectives? Thèse de doctorat en géographie, Université Paris 1 Panthéon-Sorbonne, 371 p.

McAuliffe, C., 2012. Graffiti or Street Art? Negotiating the Moral Geographies of the Creative City. Journal of Urban Affairs, $\mathrm{n}^{\circ} 34$ (2), p. 189-206. DOI : 10.1111/j.1467-9906.2012.00610.x

Sallenave L., 2017. Le « Grenoble Street Art Fes » catalyseur d'images institutionnalisées et détournées. Enjeux discursifs et territoriaux. Urbanités [en ligne], $\mathrm{n}^{\circ} 9$, mis en ligne le 29 septembre 2017, consulté le 2 février 2018. URL : http://www.revue-urbanites.fr/9-legrenoble-street-art-fest-catalyseur-dimages-institutionnalisees-et-detournees-enjeux-discursifset-territoriaux/

Valin J., 2017. Esthétique propre. La mise en administration des graffitis à Paris de 1977 à 2017. Thèse de doctorat en sciences politiques, Université Lyon 2. 


\section{NOTES}

1. «Le street art envahit la ville », Presse Océan Saint-Nazaire, 23 août 2017.

2. «Le street art fait danser les murs de la ville». Saint-Nazaire Agglomération Tourisme, http:// www.saint-nazaire-tourisme.com/content/street-art-saint-nazaire, consulté le 2 février 2018.

3. Julie Vaslin analyse le graffiti auquel elle donne toutefois une définition large qui inclut toute inscription murale inopinée perturbant l'ordre esthétique de l'espace public tel que produit par les acteurs du gouvernement urbain. Cette approche se rapproche des éléments de définition du street art proposés ci-après.

4. Voir l'appel à contribution "Les valorisations territoriales et touristiques du street art ", Echogéo. URL : http://journals.openedition.org/echogeo/15120, consulté le 2 février 2018.

5. L'artiste justifie son emploi de "graffiti» pour définir les Oides, qui se distinguent des lettrages habituellement désignés par ce terme, par la similitude des outils utilisés (le spray) ainsi que par sa volonté de rester fidèle à « l'esprit du graffiti ». Il reconnaît néanmoins que, pour une partie du public, sa série relève davantage d'un street art compris comme plus consensuel et plus officiellement valorisé, illustrant là encore les difficultés auxquelles se heurtent les tentatives de catégorisation.

6. Le questionnaire est accessible à l'adresse suivante: https://bit.ly/2J7HMb9. Ont été prises en compte l'ensemble des réponses soumises entre le 5 et le 27 décembre 2017. Conçu pour les besoins de la présente étude, ce questionnaire n'a fait l'objet d'aucune commande ni modification de la part de l'artiste.

7. L'artiste peut être contacté via une adresse électronique spécifiquement dédiée au projet, mais reste néanmoins anonyme.

8. Voir la carte "Street art in Saint-Nazaire» proposée par Saint-Nazaire Agglomération Tourisme. URL : https://bit.ly/2LiCNVW, consultée le 3 juin 2018.

9. Les Oides tirent leur nom du verlan de « doigt », en référence à la forme des personnages.

10. Page Facebook @LesOides, https://www.facebook.com/LesOides/?ref=page_internal, consultée le 30 mai 2018.

11. Compte Instagram @les_oides, https://www.instagram.com/les_oides/, consultée le 30 mai 2018.

12. https://bit.ly/2xAPSb9, consultée le 2 juin 2018.

13. 58 réponses ont été obtenues à la question invitant à préciser la nature de l'influence des Oides sur l'image qu'ont les répondants de Saint-Nazaire.

14. Plusieurs questions portaient sur une éventuelle influence des Oides sur les pratiques des répondants. Si $23 \%$ des 160 répondants déclarent une influence du projet sur leurs pratiques, les $19 \%$ qui précisent la nature de cette influence ne correspondent pas toujours aux mêmes personnes. Par ailleurs, 41 \% des 160 répondants déclarent s'être rendus grâce aux Oides dans des lieux habituellement non fréquentés, ce qui constitue de fait une forme de modification des pratiques qui n'est cependant pas identifiée comme telle par une partie des personnes interrogées. L'échantillon mobilisé sur ce point est donc variable.

\section{ABSTRACTS}

Drawing on an analysis of the Saint-Nazaire based project, Les Oides, this paper aims to address geographical and political issues related to the urban valorization of street art. It first argues that 
the artistic project can trigger evolutions of city users' representations and practices without being subject to any institutional recognition. The paper then focuses on the logic of the project's legitimization and shows that institutional valorization faces difficulties even though the project seems to be consensual. It eventually outlines the gap between street art's ability to valorize urban space on the one hand and political choices that leads to official recognition of this potential.

À partir d'une analyse du projet des «Oides" à Saint-Nazaire (Loire-Atlantique), cet article interroge les enjeux géographiques et politiques de la valorisation urbaine du street art. Il montre d'abord comment le projet artistique, indépendamment de toute reconnaissance institutionnelle, constitue un facteur d'évolution des représentations et des pratiques des usagers de l'espace urbain dans lequel il s'inscrit. Il s'intéresse ensuite aux acteurs, aux critères et aux logiques qui interviennent dans le processus de légitimation d'un projet apparemment consensuel mais dont la valorisation institutionnelle s'avère délicate, mettant ainsi en évidence l'écart entre la capacité $\mathrm{du}$ street art à valoriser un territoire et les choix auxquels répond la décision politique de reconnaître ou non ce potentiel sous la forme d'une institutionnalisation.

\section{INDEX}

Mots-clés: street art, géographie urbaine, géographie culturelle, représentation spatiale, pratique spatiale, politique culturelle, Saint-Nazaire

Keywords: street art, urban geography, cultural geography, spatial representation, spatial practice, cultural politic, Saint-Nazaire

\section{AUTHOR}

\section{AUDE LE GALLOU}

Aude Le Gallou, alegallou@outlook.fr, est Doctorante contractuelle à l' Université Paris 1 Panthéon-Sorbonne, membre de EA EIREST. Elle a publié récemment :

- Le Gallou A., 2018. From urban exploration to ruin tourism: a geographical analysis of contemporary ruins as new frontiers for urban tourism. International Journal of Tourism Cities, DOI: https://doi.org/10.1108/IJTC-12-2017-0085

- Le Gallou A., 2015. De Berlin-Est au « Nouveau Berlin » : les mémoires plurielles d'une capitale réunifiée. Géocarrefour, 90/2 | 2015, p. 153-162.

- Chilaud F., Delassalle M., Le Gallou A. et Guinard P., 2013. Los Angeles dans Mulholland Drive de David Lynch. Amerika [En ligne], 9 | 2013, mis en ligne le 20 décembre 2013, URL : http:// amerika.revues.org/4373 ; DOI : 10.4000/amerika.4373 